\title{
Assessment of cognitive impairment in long-term oxygen therapy-dependent COPD patients
}

\author{
This article was published in the following Dove Press journal: \\ International Journal of COPD \\ 29 September 2015 \\ Number of times this article has been viewed
}

\author{
Harun Karamanli ${ }^{1}$ \\ Faik Ilik ${ }^{2}$ \\ Fatih Kayhan ${ }^{3}$ \\ Ahmet Cemal Pazarli ${ }^{4}$ \\ 'Department of Pulmonology, \\ ${ }^{2}$ Department of Neurology, \\ ${ }^{3}$ Department of Psychiatry, Faculty of \\ Medicine, Mevlana University, Konya, \\ Turkey; ${ }^{4}$ Department of Pulmonology, \\ Elbistan State Hospital, Elbistan, \\ Turkey
}

Background: A number of studies have shown that COPD, particularly in its later and more severe stages, is associated with various cognitive deficits. Thus, the primary goal of the present study was to elucidate the extent of cognitive impairment in patients with long-term oxygen therapy-dependent (LTOTD) COPD. In addition, this study aimed to determine the effectiveness of two cognitive screening tests, the Mini-Mental State Examination (MMSE) and the Montreal Cognitive Assessment (MoCA), for COPD patients and the ability of oxygen therapy to mitigate COPD-related deficits in cognitive function.

Methods: The present study enrolled 45 subjects: 24 nonuser and 21 regular-user LTOTDCOPD patients. All subjects had a similar grade of education, and there were no significant differences regarding age or sex. The MoCA (cutoff: $<26$ points) and MMSE (cutoff: $\leq 24$ points) scores were compared between these two groups.

Results: The nonuser LTOTD-COPD group had a significantly lower MoCA score than that of the regular-user LTOTD-COPD group (19.38 \pm 2.99 vs $21.68 \pm 2.14$, respectively) as well as a significantly lower MMSE score. Moreover, the absence of supplemental oxygen therapy increased the risk of cognitive impairment (MoCA, $P=0.007$ and MMSE, $P=0.014$ ), and the MoCA and MMSE scores significantly correlated with the number of emergency admissions and the number of hospitalizations in the last year.

Conclusion: In the present study, the nonuser LTOTD-COPD group exhibited a significant decrease in cognitive status compared with the regular-user LTOTD-COPD group. This suggests that the assessment of cognitive function in nonuser LTOTD-COPD patients and the use of protective strategies, such as continuous supplemental oxygen treatment, should be considered during the management of COPD in this population. In addition, the MoCA score was superior to the MMSE score for the determination of cognitive impairment in the nonuser LTOTD-COPD patients.

Keywords: COPD, long-term oxygen therapy-dependent, cognitive impairment

\section{Introduction}

Although COPD is typically associated with alterations in lung function, it is a complex multicomponent disorder that is related to an extensive variety of comorbidities. COPD is commonly associated with detrimental effects in distant organs with varying degrees of severity in individual patients, but the underlying mechanisms that result in the systemic clinical manifestations and various comorbidities remain poorly understood. However, these mechanisms are likely to be associated with chronic hypoxia. ${ }^{1}$

Several studies have found that COPD patients exhibit varying degrees of cognitive impairment $(\mathrm{CI}),{ }^{2,3}$ which is one of the most common extrapulmonary symptoms of COPD ${ }^{4}$ Furthermore, $\mathrm{CI}$ is related to higher rates of mortality and disability, ${ }^{3}$ particularly in patients with severe COPD. For example, $42 \%$ of COPD patients in a nocturnal
Correspondence: Harun Karamanli Department of Pulmonology, Faculty of Medicine, Mevlana University, Aksinne Neighborhood, Esmetas Street, Number 16, 42040, Meram, Konya, Turkey

Tel +90332322 9410

Fax +903323229419

Email drharun@hotmail.com 
oxygen therapy trial exhibited clinically serious CI. ${ }^{5}$ Various types of cognitive dysfunction are correlated with a weak response to treatment and reliance on external assistance to perform basic activities of daily life. ${ }^{6}$ In theory, cognitive dysfunction could lead to deficits in daily activities that require a healthy memory, such as taking one's medication. ${ }^{7}$ Thus, the effects of COPD on behavior, particularly behaviors involving cognitive function, increases the risks associated with the disease and hence strategies for managing the disease. This is particularly important because the cognitive effects of COPD may increase medical costs and negatively impact the workforce. Therefore, COPD-related CI could have serious clinical and health care implications.

The brain may be particularly susceptible to the systemic effects of COPD. In 1992, Grant et $\mathrm{al}^{8}$ found that $42 \%$ of COPD patients had moderate to severe CI, whereas only $14 \%$ of controls exhibited CI. It is possible that the drop in arterial oxygen content, associated with COPD, may result in decreased oxygen distribution to the brain. The neurometabolic and perfusion evidences in COPD complicated by hypoxemia are coherent with an extensive subcortical and cortical hypometabolism, ${ }^{9}$ with the experimental proof that the myelinic subcortical substance is extremely vulnerable to the impact of hypoxia. ${ }^{10} \mathrm{CI}$ in COPD patients seems to be mediated by neuronal damage resulting from hypoxia and oxidative stress caused by pulmonary dysfunction or behavioral comorbidities that negatively influence the brain, such as smoking. ${ }^{5}$ It has been known for decades that there is a relationship between cognitive dysfunction and hypoxemia in patients with severe COPD ${ }^{8}$ however, it is often underappreciated. Montreal Cognitive Assessment (MoCA) test is an effective measure for screening, diagnosing, and tracking CI. ${ }^{11}$ MMSE (Mini-Mental State Examination), which has been proven to be useful and sufficient for cognitive measurements, ${ }^{12}$ provides a broad scale and does separate the specific cognitive functions. Thus, the primary aims of the present study were to evaluate the impact of acute oxygen treatment on CI in long-term oxygen therapy-dependent (LTOTD) COPD patients using the MoCA and MMSE as well as to compare the efficacy of these two measures.

\section{Materials and methods}

The Ethics Committee of Mevlana University Hospital approved the study. Informed consent was obtained from all patients prior to participation in the study. All COPD patients underwent extensive neuropsychological testing conducted by a neurologist according to standard practice.

\section{Neuropsychological assessments}

In this study, MoCA and MMSE were performed for all the patients by a neurology doctor. The patients were asked in their native language (Turkish-translated version) by exact conversion of the questions of MoCA and MMSE International Version in English. Tests were performed in randomized form in one session for all patients. The two screening scales were administered in a counterbalanced order.

\section{MoCA}

The MoCA is a 30-point test that assesses several cognitive domains and takes 10-15 minutes to complete. Mild CI is indicated by total scores $<26$ on the following subscales: visuospatial capabilities, multiple aspects of executive function, language, short-term memory, attention, concentration, and working memory.

\section{MMSE}

The MMSE, which is the most commonly used screening tool for CI, is a questionnaire comprising items that assess cognitive functioning in adults. ${ }^{13}$ It was developed in 1975 by Folstein et $\mathrm{a}^{14}$ and was initially used to evaluate elderly patients who were only capable of participating with an examiner at an optimum level for a short period of time. This 11-item instrument evaluates orientation, recall ability, short-term memory, and arithmetic ability. Each correct answer to a question scores 1 point; the total score ranges from 0 to 30 , with scores $<24$ indicating mild CI. In this study, the MMSE was administered to all the subjects by the same neurologist.

\section{Subjects}

All subjects were recruited from the patients treated at the Department of Pulmonology at Mevlana University Hospital from June 1 to December 15, 2014. They were divided into two groups: those who did not use oxygen (nonuser LTOTD-COPD group) and those who used oxygen regularly (regular-user LTOTD-COPD group). Regular-user LTOTDCOPD group was receiving continuous oxygen therapy (24 h/day) at home via nasal prongs adequate to maintain oxygen saturation between $88 \%$ and $95 \%$ from the start of oxyhemoglobin desaturation. At the beginning of the study, 24 nonuser and 21 regular-user LTOTD-COPD patients were enrolled. Only patients with LTOTD, arterial oxygen tension $\left(\mathrm{PaO}_{2}\right) \leq 55 \mathrm{mmHg}$, were included in the present study because $\mathrm{CI}$ is highly prevalent among hypoxemic COPD patients, ${ }^{9}$ and the goal of the present study was to determine the effects of 
single oxygen treatment on cognitive function. COPD patients classified as Global Initiative for Chronic Obstructive Lung Disease (GOLD) class D were selected because the study ${ }^{9}$ mentioned that adults with COPD have an increased rate of CI, particularly those who require oxygen therapy.

The inclusion criteria required that a patient be older than 40 years of age, a stable COPD patient (GOLD class D), and oxygen-dependent without the exacerbation of respiratory system for the past 2 months. All the patients were being treated with combination medical therapy consisting of long-acting beta agonists, inhaler steroids, and long-acting anticholinergic agents. The exclusion criteria included: being younger than 40 years of age; illiterate; alcoholic; obese; having severe cardiovascular comorbidities (New York Heart Association Functional Classification III/IV, myocardial infarction, or congestive heart failure), cancer, uncontrolled diabetes, or hypertension; the use of any drugs that might affect cognitive performance (eg, lithium, propanol, erythromycin antihistamine, cimetidine, or sedative agents); a history of any traumatic lesion that could affect auditory function or the central nervous system; major CI; and/or histories of head injury, brain tumor, epilepsy, dementia, or psychiatric disorders (eg, anxiety, depression, disorders, and schizophrenia).

\section{Arterial blood gas analysis}

An arterial blood gas analysis was performed on all the patients using a blood sample obtained from the radial artery while the patients breathed room air in the supine position and using oxygen. The $\mathrm{PaO}_{2}$, arterial carbon dioxide tension $\left(\mathrm{PaCO}_{2}\right)$, and blood oxygen saturation $\left(\mathrm{SO}_{2}\right)$ levels were assessed for all the subjects while breathing ambient air in supine position. All the patients were receiving continuous oxygen therapy for more than 5 years.

\section{Assessment of pulmonary physiology}

All the COPD patients met the American Thoracic Society and European Respiratory Society criteria for COPD risk classification. The regular-user LTOTD-COPD group $(n=21)$ lived in the same community as the study sample, and the two groups had similar mean ages (69.3 \pm 10 years); sex and education levels were also similar between the groups (Table 1). Standardized pulmonary function tests were performed using a dry spirometer Zan-100 spire device (nSpire Health GmbH, Oberthulba, Germany). The forced vital capacity (FVC), forced expiratory volume in 1 second $\left(\mathrm{FEV}_{1}\right)$, and $\mathrm{FEV}_{1} /$ FVC ratio were obtained for each patient after they inhaled salbutamol (Ventolin; GlaxoSmithKline plc, London, UK). The research physicians confirmed the diagnosis of COPD by spirometry outcomes $\left(\mathrm{FEV}_{1} / \mathrm{FVC}<0.7\right.$ and $\mathrm{FEV}_{1}<80 \%$ of predicted). Postbronchodilator increase in $\mathrm{FEV}_{1}$ was less than $200 \mathrm{~mL}$ and not more than $12 \%$ of the baseline value. The classification of airflow limitation severity in COPD patients was based on postbronchodilator $\mathrm{FEV}_{1}$, as defined in GOLD guidelines:

1. Mild: postbronchodilator $\mathrm{FEV}_{1} \geq 80 \%$ predicted

2. Moderate: postbronchodilator $\mathrm{FEV}_{1} \geq 50 \%$ but $<80 \%$ predicted

Table I Demographic characteristics of study subjects

\begin{tabular}{llll}
\hline & Regular-user LTOTD-COPD group & Nonuser LTOTD-COPD group & P-value \\
\hline Age (years) & $69.3 \pm 10.0$ & $68.4 \pm 14.0$ & $>0.05$ \\
Sex (M/F), $\mathrm{n}$ & $15 / 6$ & $13 / 1 \mathrm{l}$ & $>0.05$ \\
Education level (ES/HS), $\mathrm{n}$ & $15 / 6$ & $19 / 5$ & $>0.05$ \\
$\mathrm{FEV}$ & $33 \pm 11.5$ & $31.7 \pm 15.0$ & $>0.05$ \\
$\mathrm{FVC}$ & $36.9 \pm 8.5$ & $32.8 \pm 15.4$ & $>0.05$ \\
$\mathrm{PH}$ & $7.4 \pm 0.5$ & $7.3 \pm 0.6$ & $>0.05$ \\
$\mathrm{PO}_{2}$ & $47.5 \pm 11.2$ & $48.0 \pm 9.8$ & $>0.05$ \\
$\mathrm{PCO}_{2}$ & $40.1 \pm 8.6$ & $45.0 \pm 11.4$ & $>0.05$ \\
$\mathrm{SO}_{2}$ (\%) & $83.2 \pm 7.9$ & $82.5 \pm 7.9$ & $>0.05$ \\
$\mathrm{PO}_{2}$ (using oxygen) & $71.0 \pm 6.8$ & $69.0 \pm 7.3$ & $>0.05$ \\
$\mathrm{PCO}_{2}$ (using oxygen) & $48.9 \pm 2.2$ & $47.6 \pm 3.1$ & $>0.05$ \\
$\mathrm{SO}_{2}$ (\%) (using oxygen) & $90.1 \pm 5.0$ & $89 \pm 6.2$ & $>0.05$ \\
Smoking (pack years) & $39.95 \pm 20.9$ & $40.12 \pm 15.1$ & $>0.05$ \\
Number of hospitalizations in last year & $1.6 \pm 1.0$ & $2.8 \pm 2.8$ & $>0.05$ \\
Number of emergency admissions in last year & $2.8 \pm 4.3$ & $5.8 \pm 10.4$ & $0.011 *$ \\
\hline
\end{tabular}

Notes: Data are presented as means \pm SD, unless otherwise specified. *There was a significant difference between the number of emergency admissions in the last year between the regular-user LTOTD-COPD group and the nonuser LTOTD-COPD group.

Abbreviations: $\mathrm{M}$, male; F, female; ES, elementary school; $\mathrm{HS}$, high school; $\mathrm{FEV}_{1}$, forced expiratory volume in I second; FVC, force vital capacity; $\mathrm{P}_{2}$, partial oxygen pressure; $\mathrm{PCO}_{2}$, partial carbon dioxide pressure; $\mathrm{SO}_{2}$, oxygen saturation; LTOTD, long-term oxygen therapy-dependent. 
3. Severe: postbronchodilator $\mathrm{FEV}_{1} \geq 30 \%$ but $<50 \%$ predicted

4. Very severe: postbronchodilator $\mathrm{FEV}_{1}<30 \%$ predicted. Subjects with $\mathrm{FEV}_{1}$ lower than $30 \%$ predicted and oxygen-dependent were classified as the LTOTD group according to GOLD 2010 criteria.

\section{Statistical analysis}

The data of the nonuser and regular-user LTOTD-COPD patients were analyzed and compared. The data were first evaluated using descriptive statistics by comparing the means of the various parameters for the regular-user LTOTD-COPD group with those for the nonuser LTOTD-COPD group using Mann-Whitney $U$-tests. Spearman's correlation analyses were performed to assess the relationships between the clinical and neuropsychological variables in each group, as appropriate. A multiple linear regression model was used to identify independent factors from the MoCA and MMSE tests that were associated with age, sex, and education level. A $P$-value less than 0.05 was considered to indicate statistical significance.

\section{Results}

The demographic characteristics of the study groups are provided in Table 1. The regular-user and nonuser LTOTDCOPD groups were statistically similar with respect to sex, age, smoking status, and the duration of formal education. Similarly, no significant differences were observed between the two groups in terms of average $\mathrm{FEV}_{1}, \mathrm{FVC}, \mathrm{pH}, \mathrm{PaO}_{2}$, $\mathrm{PaCO}_{2}$, or $\mathrm{SO}_{2}$ (Table 1).

The reasons why the nonuser LTOTD-COPD patients were not receiving oxygen therapy are shown in Table 2.

All the COPD patients were classified as stage IV (high severity of airflow obstruction) according to the GOLD classification. The MoCA ( $P=0.007$; Figure 1$)$ and MMSE $(P=0.014$; Figure 2$)$ scores of the nonuser LTOTD-COPD group were significantly lower than those of the regular-user LTOTD-COPD group (Table 3).

Table 2 Reasons for poor compliance among nonuser LTOTDCOPD patients

\begin{tabular}{lll}
\hline Reasons & Frequency & Percentage \\
\hline Financial problems causing difficulty & 8 & 33.3 \\
in paying for electricity & & \\
Headache & 10 & 41.6 \\
Machine is noisy when operating & 2 & 8.3 \\
Machine breakdown & 2 & 8.3 \\
Not convinced of benefit of & 2 & 8.3 \\
oxygen therapy & & \\
Total & 24 & 100 \\
\hline
\end{tabular}

Abbreviation: LTOTD, long-term oxygen therapy-dependent.

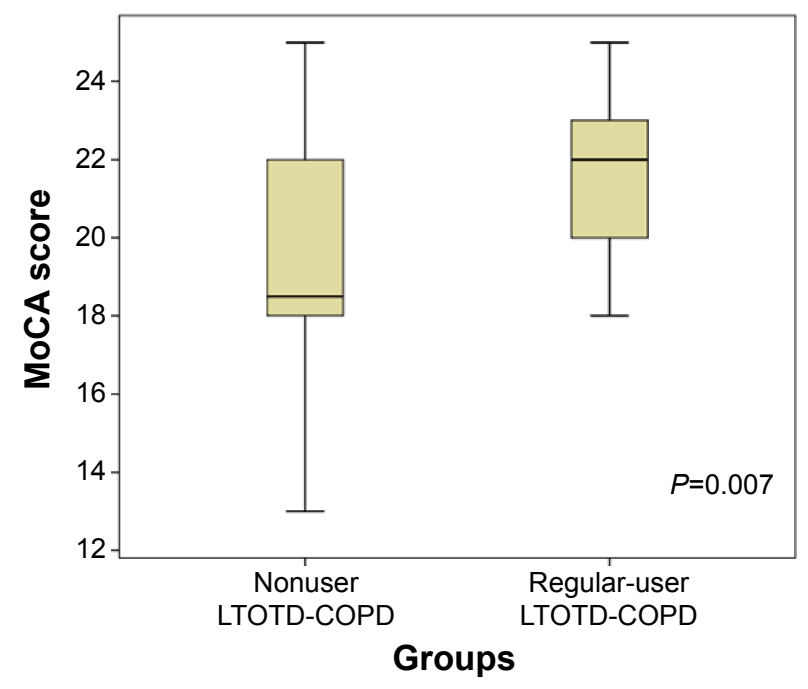

Figure I Box plot of the MoCA scores for the two study groups. Abbreviations: LTOTD, long-term oxygen therapy-dependent; MoCA, Montreal Cognitive Assessment.

The MoCA score was negatively correlated with the number of hospitalizations $(r=-0.57 ; P<0.0001)$ and the number of emergency admissions in the last year $(r=-0.39$; $P=0.01$ ) in both LTOTD-COPD groups (Table 4). Similarly, the MMSE score was negatively correlated with the number of hospitalizations $(r=-0.52 ; P<0.0001)$ and number of emergency admissions in the last year $(r=-0.40 ; P=0.007)$ in both LTOTD-COPD groups. In addition, the MMSE score was negatively correlated with age $(r=-0.49 ; P=0.001$; Table 5$)$.

\section{Discussion}

The present findings revealed a significant overall deficit in cognitive functioning among the nonuser LTOTD-COPD

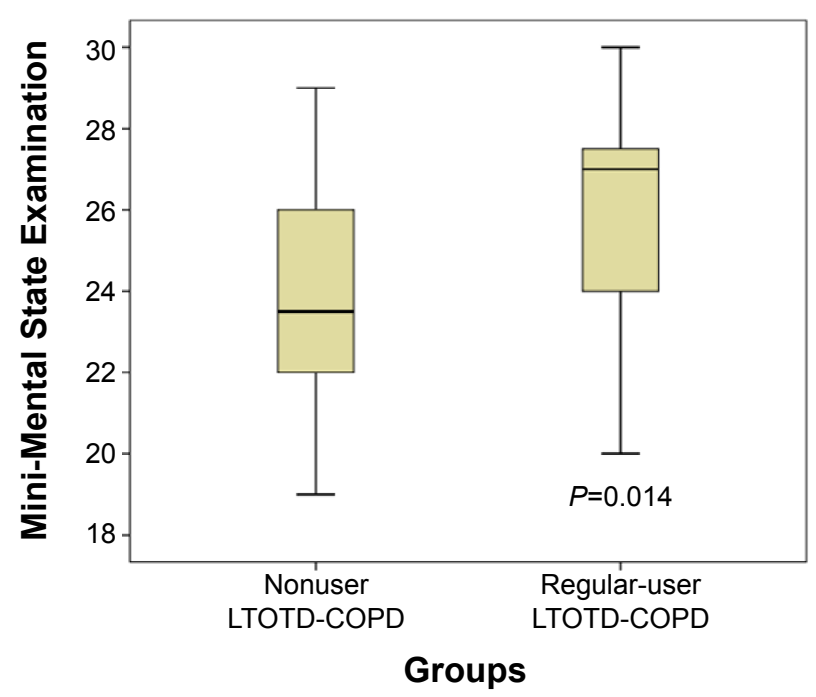

Figure 2 Box plot of the MMSE scores for the two study groups. Abbreviations: LTOTD, long-term oxygen therapy-dependent; MMSE, MiniMental State Examination. 
Table 3 The proportion of patients with cognitive impairment

\begin{tabular}{lll}
\hline Groups & MoCA (+), \% & MMSE (+), \% \\
\hline Regular-user LTOTD-COPD group & $36.8 \%$ & $26.3 \%$ \\
Nonuser LTOTD-COPD group & $70.8 \%$ & $50 \%$ \\
\hline
\end{tabular}

Note: "+" indicates cognitive assessment test result is positive for cognitive impairment.

Abbreviations: LTOTD, long-term oxygen therapy-dependent; MMSE, MiniMental State Examination; MoCA, Montreal Cognitive Assessment.

group after controlling for confounding factors known to influence cognitive abilities, such as age, sex, smoking, and education level. ${ }^{15}$ In addition, the present results indicated that the global cognitive functions of the nonuser LTOTDCOPD group were worse than those of the regular-user LTOTD-COPD group. Attention, memory, and executive functions are often reported as the most commonly impaired cognitive domains in patients with LTOTD-COPD in MoCA test, whereas cognitive decline on the word list recall, animal category fluency, and symbol digit modalities are the commonly impaired cognitive domains in MMSE test. This study is the first to demonstrate an association between cognitive decline in nonuser LTOTD-COPD patients and oxygen use. These findings provide evidence that will allow physicians to take effective measures for the prevention and treatment of cognitive decline in this patient group. Resting hypoxemia is a crucial risk factor for CI, and a treatment regimen that includes ineffective supplemental oxygen markedly increases this risk. Due to lower sensitivity of the MMSE, ${ }^{16}$ the MoCA was thought to be a more appropriate tool for the assessment of cognitive function for patients in this study. A comparative analysis of the MoCA and MMSE determined that the MoCA was superior to the MMSE in detecting CI in at-risk populations ${ }^{16}$ To our knowledge, this is the first study to employ the MoCA questionnaire to evaluate cognitive functioning in nonuser LTOTD-COPD patients. The primary finding of this study was that nonuser LTOTD-COPD patients were at a higher risk for CI compared with regular-user LTOTD-COPD patients. The results of this study suggest that the patient group with nonuser LTOTD-COPD subjects shows significant CI. Our study's results showed a strong correlation of cognitive decline in COPD subjects.

Early studies initially investigated cognitive functioning in severe COPD patients with hypoxemia. ${ }^{17}$ As early as 1996 , studies demonstrated that adults with COPD, particularly those who required oxygen therapy, showed more frequent occurrences of $\mathrm{CI} .{ }^{17} \mathrm{~A}$ prolonged oxygen deprivation during everyday activities is an important causative mechanism underlying permanent diffuse injury to brain tissue. ${ }^{18}$ A study has proposed that intermittent and continuous hypoxia resulting from poor lung function in COPD patients may cause temporary deficits in the metabolism of neurotransmitters in the central nervous system. ${ }^{19}$ In hypoxic situations, cerebral blood perfusion decreases, which may increase ischemia in some brain areas, perhaps resulting in subcortical atrophy. ${ }^{9}$ Hypoxic episodes or chronic hypoxia in the brain can also lead to the production of free radicals, an increase in inflammationmediated neurotoxic effects, and alterations in the activity of oxygen-dependent enzymes that mediate neuronal damage. ${ }^{20}$ Moreover, it has been known for 30 years that long-term oxygen supplementation in COPD patients with hypoxemia may have a beneficial influence on cognitive function. ${ }^{21}$ The presence of CI was identified in $77 \%$ of patients with comorbid COPD and hypoxemia. ${ }^{8}$ Mental and cognitive dysfunction can be effectively identified using oxyhemoglobin desaturation levels, ${ }^{22}$ and 6 months of oxygen treatment in patients with hypoxemic COPD was associated with small but significant improvements in brain function. ${ }^{5}$

A number of studies have used the MMSE to assess cognitive dysfunction, but have produced variable results. For example, although patients with severe COPD exhibit significant impairment on the MMSE, ${ }^{17}$ LTOTD-COPD patients with severe hypoxemia do not. Thus, based on the present findings, it appears that the MoCA test is a more sensitive and reliable screening tool than the MMSE, at least for the evaluation of hypoxemia-related chronic central nervous system deficits in patients with LTOTD-COPD. The results of this study also demonstrated that MoCA was more sensitive than MMSE when assessing cognitive functioning in patients with severe hypoxemia, because there was a statistical significance between MoCA's $P$-value and MMSE's $P$-value in our study.

Although a majority of previous studies have included patients with moderate-to-severe as well as nonhypoxemic COPD, they assessed only a LTOTD-COPD patient population. ${ }^{9}$ To our knowledge, this is the first study to investigate the effects of chronic hypoxemia on cognitive

Table 4 Mean values of MoCA and MMSE scores for our study groups

\begin{tabular}{llll}
\hline Cognitive test & Nonuser LTOTD-COPD & Regular-user LTOTD-COPD & P-value \\
\hline MoCA & $19.37 \pm 2.99$ & $21.68 \pm 2.14$ & 0.007 \\
MMSE & $23.75 \pm 2.63$ & $25.89 \pm 2.79$ & 0.014 \\
\hline
\end{tabular}

Notes: Data are presented as means \pm SD. P-values were calculated by Mann-Whitney U-tests.

Abbreviations: LTOTD, long-term oxygen therapy-dependent; MoCA, Montreal Cognitive Assessment; MMSE, Mini-Mental State Examination. 
Table 5 Correlation between MoCA-MMSE score and clinical parameters

\begin{tabular}{lll}
\hline $\begin{array}{l}\text { Cognitive } \\
\text { test }\end{array}$ & $\begin{array}{l}\text { Number of } \\
\text { hospitalizations } \\
\text { in last year }\end{array}$ & $\begin{array}{l}\text { Number of emergency } \\
\text { admissions in last year }\end{array}$ \\
\hline MoCA & & \\
$\quad$ Correlation & -0.57 & -0.39 \\
$\quad \begin{array}{l}\text {-value } \\
\text { MMSE }\end{array}$ & $<0.0001$ & 0.01 \\
$\quad$ Correlation & -0.52 & -0.40 \\
$\quad P$-value & $<0.0001$ & 0.007 \\
\hline
\end{tabular}

Note: $P$-values calculated with Mann-Whitney U-test.

Abbreviations: MoCA, Montreal Cognitive Assessment; MMSE, Mini-Mental State Examination.

functioning in stable LTOTD-COPD patients using both MoCA and MMSE. The present findings suggest that nonuser LTOTD-COPD patients exhibit significant cognitive and functional impairments compared with LTOTD-COPD patients who use regular oxygen treatment. Based on pathological considerations, it was hypothesized that MoCA and MMSE would be sensitive to changes in several cognitive domains in these patients. These findings indicate that it is important to continually treat oxygen-poor states in patients with LTOTDCOPD to avoid exacerbation of CI. Relationship between the cognitive function and hypoxemia has been further supported by the fact that MoCA and MMSE scores in this study significantly correlated with the nonuser LTOTD-COPD group.

Based on the scores of the MoCA and the MMSE, the present study demonstrated an increase in cognitive impairment in nonuser LTOTD-COPD patients. However, the impact of COPD on cognitive performance is partially reversible via oxygen therapy and physical activity, although this is often underappreciated. ${ }^{13}$ If COPD patients do not appreciate the extent to which oxygen treatment is beneficial in terms of cognitive function and therefore fail to use oxygen supplementation, they may experience a variety of cognitive deficits identified by a previous study: cognitive dysfunction seriously impacts self-management and can lead to inappropriate and incorrect use of, particularly, medicinal inhalants, ${ }^{23}$ and thus poor adherence to medication increases the risk of an acute exacerbation. ${ }^{4}$ Also, untreated cognitive difficulties have a considerable effect on COPD patients and may have an additive impact on respiratory-related hospital admissions and mortality. ${ }^{23}$

The acute exacerbations of COPD symptoms that occur during the course of the disease may impair one's quality of life, contribute to the deterioration of spirometric tests, and increase mortality rates. COPD patients perform worse on cognitive function tests than age-matched controls, and a significant correlation has been shown between the number of hospitalizations and quality of life in COPD patients. ${ }^{24}$ Although COPD patients experiencing an acute exacerbation were not included in this study, a number of these patients have been assessed in the last year. Preliminary data suggest meaningful correlations of cognitive functioning with the number of hospitalizations and the number of emergency admissions in the last year. In addition, during symptom exacerbation, hypoxemia, systemic inflammation, and medication use tend to increase, which can also contribute to the cognitive decline of these patients. It is also possible that these patients experience increased systemic inflammation and probable hypoxic conditions that affect the cognitive functioning of these patients, or that these patients experience more frequent acute exacerbations due to difficulties managing medical treatment regimens or pulmonary rehabilitation programs.

Patients with obesity were excluded in our study, because obesity is known to be the most important risk factor that leads to CI. Obesity is a factor that can cause systemic inflammation. It can destroy the integrity of the brain-blood barrier, permitting irritants to enter the brain and induce the production of inflammatory cytokines. The cytokines disrupt synaptic transmission across the neurons and neurogenesis, and some inflammatory cytokines damage the surrounding neurons by stimulating apoptosis. Thus, the destruction of the surrounding neurons leads to decrease in cognitive function.

In this study, we observed overall significant cognitive deficits in LTOTD-COPD patients after we controlled for confounding factors, including education, smoking, and body mass index (BMI), known to affect cognition. Notably, education is a significant determinant of $\mathrm{CI} \cdot{ }^{25}$ Education and $\mathrm{CI}$ are intensely interrelated. Unfortunately, we were unable to establish the precise role that patient's education level plays in the frequency of $\mathrm{CI}$ in this study. Because in our study, we tried to diminish the effect of this variable using normative data adjusted for education. Furthermore, nonuser LTOTD-COPD group and regular-user LTOTD-COPD group were well matched for education. For that reason, these factors unlikely account for the higher frequency of CI found in nonuser LTOTD-COPD group than in regular-user LTOTD-COPD group.

\section{Limitations}

The present study includes several limitations. First, the study population was small, which may account, at least in part, for the weak correlation between some of the measures in the present outcome analyses. Second, it was impossible to accurately determine compliance with their past treatment 
regimens or the history of confounding factors such as work and marital status. Although the participants of this study were recruited based on stringent inclusion criteria, it is possible that alternative or subchronic diseases may have also played a role in the CI of these COPD patients.

\section{Conclusion}

In conclusion, despite these limitations, this study has several strengths. First, this study highlights the similarities of the MoCA and MMSE and describes their use in the LTOTDCOPD patient population. Second, due to its own limitations and sensitivity to confounding factors such as age, the MMSE cannot be considered a gold standard test in studies using a prospective clinical and neuropsychological study design. Thus, the use of the MoCA for the assessment of cognitive function in LTOTD-COPD patients was considered more appropriate.

The cognitive functioning of nonuser LTOTD-COPD patients was adversely affected by hypoxia, and the MoCA proved a valuable instrument for the identification of $\mathrm{CI}$ in LTOTD-COPD patients. Moreover, clinicians should continually assess their LTOTD-COPD patients for CI in order to determine which patients may benefit from the regular use of supplemental oxygen therapy.

\section{Author contributions}

Drs Karamanli, Ilik, and Kayhan are guarantors of the article.

Dr Karamanli contributed to data acquisition, analysis, and interpretation; wrote the first draft of the manuscript; and revised it following critical reviews by the coauthors. Dr Ilik contributed to study design, data acquisition and interpretation, study coordination, and critically reviewed the manuscript. Dr Kayhan contributed to data acquisition and critically reviewed the manuscript. Dr Pazarli contributed to data acquisition, analysis, and interpretation. All authors contributed toward data analysis, drafting and critically revising the paper, and agree to be accountable for all aspects of the work.

\section{Disclosure}

The authors declare that they have no conflicts of interest regarding this paper.

\section{References}

1. Barnes PJ. Chronic obstructive pulmonary disease: effects beyond the lungs. PLoS Med. 2010;7(3):e1000220.

2. Antonelli-Incalzi R, Corsonello A, Trojano L, et al. Screening of cognitive impairment in chronic obstructive pulmonary disease. Dement Geriatr Cogn Disord. 2007;23(4):264-270.
3. Antonelli-Incalzi R, Corsonello A, Pedone C, et al. Drawing impairment predicts mortality in severe COPD. Chest. 2006;130(6):1687-1694.

4. Dodd JW, Getov SV, Jones PW. Cognitive function in COPD. Eur Respir J. 2010;35(4):913-922.

5. Heaton RK, Grant I, McSweeny AJ, Adams KM, Petty TL. Psychologic effects of continuous and nocturnal oxygen therapy in hypoxemic chronic obstructive pulmonary disease. Arch Intern Med. 1983;143(10): 1941-1947.

6. Incalzi RA, Gemma A, Marra C, Capparella O, Fuso L, Carbonin P Verbal memory impairment in COPD: its mechanisms and clinical relevance. Chest. 1997;112(6):1506-1513.

7. Orsitto G, Cascavilla L, Franceschi M, et al. Influence of cognitive impairment and comorbidity on disability in hospitalized elderly patients. J Nutr Health Aging. 2005;9(3):194-198.

8. Grant I, Heaton RK, McSweeny AJ, Adams KM, Timms RM. Neuropsychologic findings in hypoxemic chronic obstructive pulmonary disease. Arch Jntern Med. 1982;142(8):1470-1476.

9. Antonelli Incalzi R, Marra C, Giordano A, et al. Cognitive impairment in chronic obstructive pulmonary disease - a neuropsychological and spect study. J Neurol. 2003;250(3):325-332.

10. Stys PK. White matter injury mechanisms. Curr Mol Med. 2004;4(2): 113-130.

11. Smith T, Gildeh N, Holmes C. The Montreal Cognitive Assessment: validity and utility in a memory clinic setting. Can J Psychiatry Revue Canadienne de Psychiatrie. 2007;52(5):329-332.

12. Ozge C, Ozge A, Unal O. Cognitive and functional deterioration in patients with severe COPD. Behav Neurol. 2006;17(2):121-130.

13. Derkacz M, Mosiewicz J, Myslinski W. [Cognitive dysfunction in patients with chronic obstructive pulmonary disease]. Wiad Lek. 2007; 60(3-4):143-147. Polish.

14. Folstein MF, Folstein SE, McHugh PR. "Mini-mental state". A practical method for grading the cognitive state of patients for the clinician. J Psychiatric Res. 1975;12(3):189-198.

15. Bawden FC, Oliveira CA, Caramelli P. Impact of obstructive sleep apnea on cognitive performance. Arq Neuropsiquiatr. 2011;69(4):585-589.

16. Dong Y, Lee WY, Basri NA, et al. The Montreal Cognitive Assessment is superior to the Mini-Mental State Examination in detecting patients at higher risk of dementia. Int Psychogeriatr. 2012;24(11):1749-1755.

17. Isoaho R, Puolijoki H, Huhti E, Laippala P, Kivela SL. Chronic obstructive pulmonary disease and cognitive impairment in the elderly. Int Psychogeriatr. 1996;8(1):113-125.

18. Borson S, Scanlan J, Friedman S, et al. Modeling the impact of COPD on the brain. Int J Chron Obstruc Pulmon Dis. 2008;3(3):429-434.

19. Gibson GE, Pulsinelli W, Blass JP, Duffy TE. Brain dysfunction in mild to moderate hypoxia. Am J Med. 1981;70(6):1247-1254.

20. Ryu CW, Jahng GH, Choi CW, et al. Microstructural change of the brain in chronic obstructive pulmonary disease: a voxel-based investigation by MRI. COPD. 2013;10(3):357-366.

21. Thakur N, Blanc PD, Julian LJ, et al. COPD and cognitive impairment: the role of hypoxemia and oxygen therapy. Int J Chron Obstruc Pulmon Dise. 2010;5:263-269.

22. Incalzi RA, Gemma A, Marra C, Muzzolon R, Capparella O, Carbonin P. Chronic obstructive pulmonary disease. An original model of cognitive decline. Am Rev Respir Dis. 1993;148(2):418-424.

23. Hung WW, Wisnivesky JP, Siu AL, Ross JS. Cognitive decline among patients with chronic obstructive pulmonary disease. Am J Respir Crit Care Med. 2009;180(2):134-137.

24. Dodd JW, Charlton RA, van den Broek MD, Jones PW. Cognitive dysfunction in patients hospitalized with acute exacerbation of COPD. Chest. 2013;144(1):119-127.

25. Sattler C, Toro P, Schönknecht P, Schröder J. Cognitive activity, education and socioeconomic status as preventive factors for mild cognitive impairment and Alzheimer's disease. Psychiatry Res. 2012;196(1): 90-95. 
International Journal of COPD

Dovepress

\section{Publish your work in this journal}

The International Journal of COPD is an international, peer-reviewed journal of therapeutics and pharmacology focusing on concise rapid reporting of clinical studies and reviews in COPD. Special focus is given to the pathophysiological processes underlying the disease, intervention programs, patient focused education, and self management protocols.

This journal is indexed on PubMed Central, MedLine and CAS. The manuscript management system is completely online and includes a very quick and fair peer-review system, which is all easy to use. Visit http://www.dovepress.com/testimonials.php to read real quotes from published authors.

Submit your manuscript here: http://www.dovepress.com/international-journal-of-chronic-obstructive-pulmonary-disease-journal 